\title{
Functional dissociation of the hippocampus and prefrontal cortex in learning and memory
}

\author{
GORDON WINOCUR \\ Trent University, Peterborough, Ontario, Canada
}

\begin{abstract}
In Experiment 1, rats with lesions to the prefrontal cortex were tested on a variable-interval delayed alternation problem, and their performance was compared with that of rats with hippocampal lesions in a previous study (Winocur, 1985). In Experiments 2 and 3, groups of prefrontal, hippocampal, and operated control rats were administered a test of conditional discrimination learning. Prefrontal rats were impaired in learning both tasks, but their performance was not affected by temporal manipulations that challenged episodic memory. In contrast, rats with hippocampal lesions were not impaired in basic learning, but their performance on both tasks deteriorated at relatively long delays. The results confirmed the importance of the hippocampus in long-term recall of specific events and implicated the prefrontal cortex in nonepisodic learning and memory functions.
\end{abstract}

The importance of the hippocampus for normal memory function is now well established. Extensive research has shown that damage to the hippocampus produces severe amnesia for specific events that occur within a defined spatiotemporal context. In contrast, memory for nonspecific information that is not tied to a particular context is relatively protected in hippocampal amnesia. Thus animals and humans with hippocampal damage frequently demonstrate memory for complex skills and rules without remembering specific features of the episodes in which such information was acquired (Milner, 1972; Squire, 1987). This pattern of lost and spared function has been taken as evidence for the existence of separate memory systems subserved by different neural mechanisms (see, e.g., Cohen \& Squire, 1980; Kesner, 1984; Olton, Becker, \& Handelmann, 1979).

Although the hippocampus and other subcortical structures (e.g. , the dorso-medial thalamus) are considered important for recalling specific events, the neural basis of nonspecific memory remains less well described. One brain region that may be implicated in nonspecific aspects of learning and memory is the prefrontal cortex. Historically, the prefrontal cortex has been identified with executive-like functions that regulate the organization of information and planning of behavior. Support for this general view includes evidence, from animal and clinical studies, that damage to the prefrontal area disrupts performance on tasks that require temporal ordering of information (Milner, 1974), response sequencing (Pribram,

This research was supported by a grant from the Natural Sciences and Engineering Research Council of Canada. I am grateful to Morris Moscovitch for useful discussions and for helpful comments on an early draft of this paper. Thanks also to Bruce Osborne, Bruce Scott, John Zomer, and Doris Pereschuk for their able technical support. Correspondence should be addressed to Gordon Winocur, Department of Psychology, Trent University, Peterborough, Ontario K9J 7B8, Canada.
1973), and the acquisition of rule-based behavior (Shallice, 1982). These tasks are complex and typically include specific and nonspecific memory components. However, there is little evidence that such deficits result from a loss of memory for the specific tasks. On the other hand, an impaired use of memory, as in a failure to apply acquired information in selecting responses or executing appropriate strategies, is a distinct possibility.

Recently, Winocur and Moscovitch (1990) compared the effects of hippocampal and prefrontal lesions in rats on complex maze performance. Both groups were impaired in original maze learning, but subsequent testing, on the same and different maze problems, revealed clear differences in their respective deficits. The rats with hippocampal lesions displayed considerable sparing of memory for the general skill of maze learning but virtually no memory for the particular maze on which they had been trained. In contrast, the prefrontal group showed good retention of the familiar maze, but failed to transfer general information about maze running to another, similarly designed, maze. The results confirmed the importance of the hippocampus for recalling highly specific information and, at the same time, pointed to a possible role for the prefrontal cortex in learning and remembering skill-related information.

This line of investigation has been extended in the present study, to determine whether a similar dissociation can be demonstrated in other tests that combine specific and nonspecific memory requirements. In Experiment 1 , rats with lesions in the prefrontal cortex were tested on a variable-interval delayed alternation task (DA) that has proved to be sensitive to a variety of manipulations, including the differential effects of localized brain damage. In previous work, rats with hippocampal lesions normally learned the basic response-alternation rule associated with this task, but their performance declined significantly when they were required to recall specific in- 
formation from the preceding trial over relatively long intervals (Winocur, 1985). On the basis of other research (Kolb, 1984), impaired alternation learning was expected in rats with prefrontal lesions, but the question of particular interest was whether or not increases in the memory load would influence the performance of these animals.

In Experiments 2 and 3, groups of rats with lesions in the prefrontal cortex or hippocampus were administered a test of conditional discrimination learning similar to that developed by Petrides $(1982,1985)$ for use with monkeys and humans. In this task, animals must learn to associate different responses with different stimuli. Petrides (1982, 1985 ) found that damage to the prefrontal cortex, but not the medial temporal lobe area, produced severe deficits in forming the conditional associations. In the present experiments, an attempt was made to reproduce these results in rats and to determine whether or not extending critical intervals between stimulus presentation and the opportunity to respond differentially would affect the lesioned groups.

\section{EXPERIMENT 1}

The DA test was conducted in an operant chamber with a single retractable lever. Essentially, the test is an alternating go/no-go task with a variable interval between the go and no-go trials. To perform successfully, rats must learn the basic alternation rule and be able to remember, on each trial, the events of the preceding trial. For example, following a go trial, the rat must recall, over the intertrial interval (ITI), that leverpresses were rewarded on that trial and determine that a no-go trial will follow in which such responses will not be rewarded.

Previous work has shown that hippocampal lesions selectively affect performance at relatively long ITIs (Winocur, 1985). In the same study, rats with lesions in the dorso-medial thalamus produced a more generalized deficit that affected their learning of the basic alternation habit, as well as specific memory at all intervals. The purpose in this experiment was to study rats with lesions in the prefrontal cortex on the DA task, and compare their behavior to that of the other lesion groups. If, in fact, the disruptive effects of prefrontal lesions are restricted to nonspecific learning and memory, a deficit was expected only on the rule-learning part of the task.

\footnotetext{
Method

Subjects. Eighteen male Long-Evans rats, weighing 350-400 g, were obtained from the Trent University Breeding Center, and served as subjects. Throughout the experiment, rats were housed individually in wire cages with water available at all times. Food was provided according to experimental conditions.

Apparatus. Testing was conducted in two identical Skinner boxes, each equipped with a single retractable lever located to the right of a central feeder. Each box was contained in a sound-proof chamber $(52.5 \times 52.5 \times 52.5 \mathrm{~cm})$. A 3-W light mounted in the ceiling of the chamber provided general illumination.

During training and during the go trials of the DA testing, each leverpress produced a single 45-mg Noyes food pellet in the central feeder. Each Skinner box was connected to its own Apple II+
}

microcomputer, which controlled all aspects of testing and recorded the latency to the first response in each trial, as well as the number of responses per trial.

Surgery and Histology. The rats were assigned in equal numbers to prefrontal (PFC) and operated control (OC) Groups. In preparation for surgery, the rats were anesthetized with sodium pentobarbital $(50 \mathrm{mg} / \mathrm{kg}$ ) and positioned in a Johnson-Krieg stereotaxic instrument. In the rats that received prefrontal lesions, a midline incision was made, and holes, approximately $5 \mathrm{~mm}$ in diameter, were drilled in the anterior skull. The dorsomedial and dorsolateral prefrontal cortex was aspirated through a 20-ga hypodermic needle. The rats prepared as operated controls had holes drilled in their skulls, but no brain tissue was removed.

After the experiment, the rats in the PFC Group were deeply anesthetized with ether and perfused through the heart with physiological saline followed by $10 \%$ formol saline. The brains were embedded with paraffin before sectioning. All sections were stained with thionin, and the extent of damage was determined by comparisons with the standard atlas of Konig and Klippel (1963).

Procedure. Of 20 rats that were subjected to surgery, 18 survived and participated in the experiment (OC group, $n=10 ; \mathrm{PFC}$ group, $n=8$ ). All rats were allowed 2 weeks to recover from surgery. They were then placed on a food-deprivation schedule that gradually reduced their body weights to $80 \%$ of normal. Throughout the experiment, the rats were maintained at a constant weight by being fed about $20 \mathrm{~g}$ of lab chow each day.

Training began about 1 month after surgery. Initially, the rats were trained to press the lever for food according to a continuous reinforcement (CRF) schedule. The rats received daily $15-\mathrm{min}$ sessions until a minimum rate of 75 responses per session was achieved over 2 consecutive days.

DA testing was initiated the day after criterion was reached on CRF training. Each session consisted of 12 reinforced (go) trials alternating with 12 nonreinforced (no-go) trials. The lever was always present during the go and no-go trials, each of which was $20 \mathrm{sec}$ long. Each leverpress during the go trials produced a food pellet, whereas leverpresses during the no-go trials were not rewarded. The go and no-go trials were separated by a variable ITI, during which the lever was retracted. ITIs were $0,2.5,5,10,20$, 40 , or $80 \mathrm{sec}$ long, with each delay occurring twice after the go trials and twice after the no-go trials, for a total of four times per session. The sequence for ITIs was varied for each session, which always began with a go trial. The rats received one session per day for 12 days.

\section{Results and Discussion}

There was no difference between groups in acquiring the leverpressing response. The PFC and OC groups both achieved stable CRF responding in six to eight sessions. Analysis of variance (ANOVA) indicated no significant difference on this measure $(F<1)$. An ANOVA was also performed on the number of responses made by each rat in the last CRF session, and, here again, there was no significant group difference $(F<1)$.

For DA testing, only the data based on latency measures will be presented. The response output data yielded essentially the same information and was considered redundant for the present purposes.

The results for test Days 1 and 12 are presented in Figure 1 in the form of go/no-go latency ratios. Ratios were calculated, at each ITI, by dividing the mean latency to the first response in the go trials by the mean latency to the first response in the no-go trials. A low ratio would result from shorter latencies in the go than in the no-go 


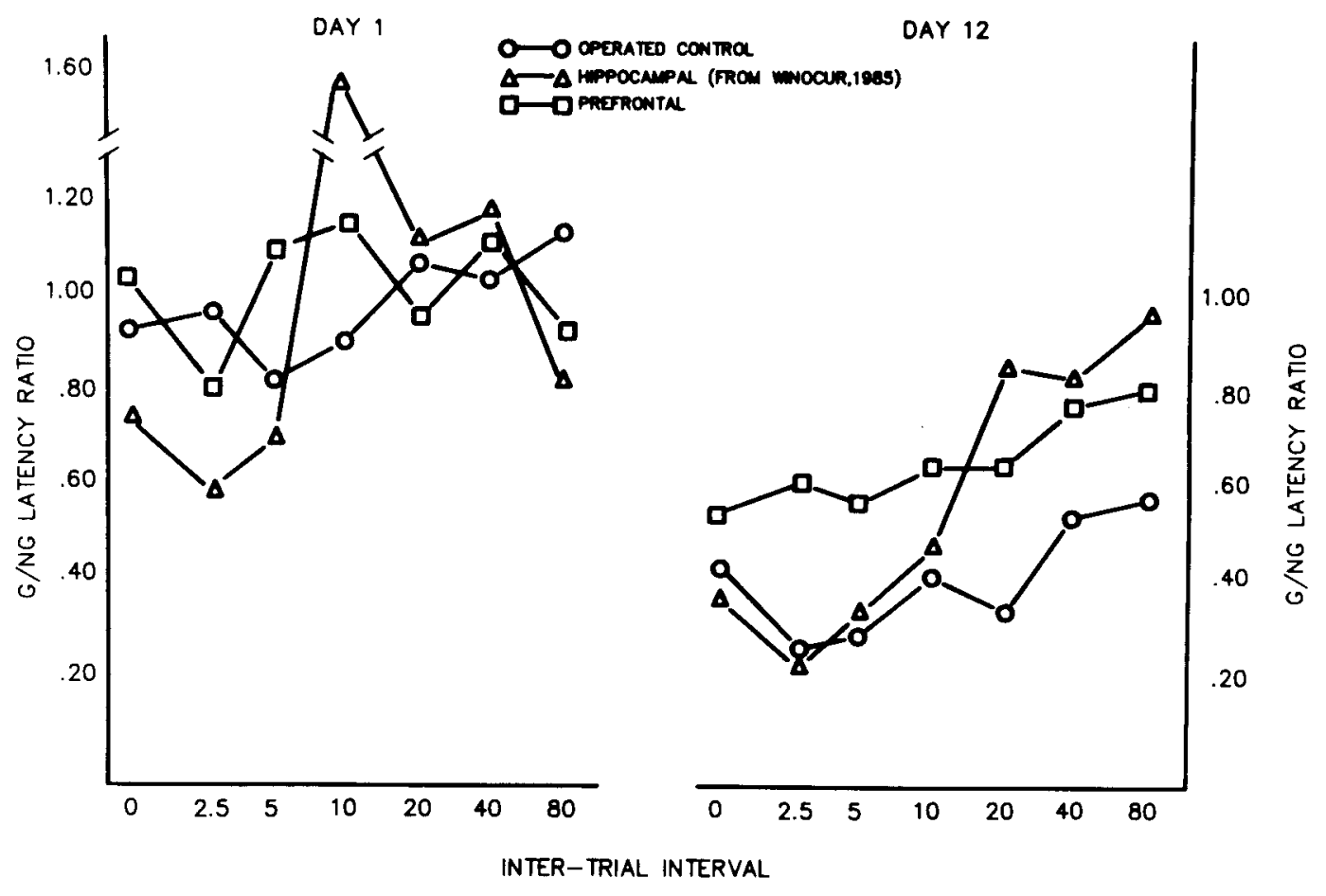

Figure 1. Mean latency ratios on Days 1 and 12 of the delayed alternation task of Experiment 1.

trials. Thus, the lower the latency ratio, the better the rat's performance. Figure 1 presents the ratios for the PFC and OC groups and, for purposes of comparison, the corresponding scores for the hippocampal group in the Winocur (1985) study are also provided. The latter group's data were not entered into the statistical analysis performed on the PFC and OC groups.

The PFC and OC groups performed inefficiently on Day 1, the day that rats were transferred from CRF training to DA testing. On Day 1, the average latency ratio over the range of ITIs for each group was approximately 1.00 , indicating that response latencies were equal in the go and no-go trials. The variations in performance that did occur were unrelated to effect of lesion or length of ITI (all $p s>.05$ ). As can be seen in Figure 1, on Day 1, neither the PFC nor the OC group behaved differently than the hippocampal group of the previous experiment.

By Day 12 , the PFC and OC groups improved, relative to their performance on Day 1 . The pattern of performance on Day 12 was similar for both groups, in that latency ratios were lower at short ITIs and increased with the length of the ITI. However, the OC group consistently performed better than the PFC group. An ANOVA applied to the Day 12 data yielded significant main effects of group $[F(1,16)=6.18, p<.025]$ and ITI $[F(6,96)=$ $15.83, p<.001]$. The group $\times$ ITI interaction $[F(6,96)=$ $1.61, p>.05]$ was not significant.

The PFC group's deficit in alternation rule learning is also revealed in Figure 2, where each group's latency ratios at the 0-sec ITI were isolated and plotted over the 12 test days. The 0 -sec ITI scores are important because, at this interval, there was virtually no delay between trials, and demands on specific memory were minimal. Successful performance at the 0-sec ITI depended on the rat's ability to implement the response-alternation rule. The data, presented in this way, show that the acquisition rate was slower in the PFC group than in the OC group. This observation was confirmed by a significant group $\times$ block interaction $[F(5,80)=2.70, p<.025]$.

By way of comparison, it can be seen that, while the PFC group and the hippocampal group of the previous

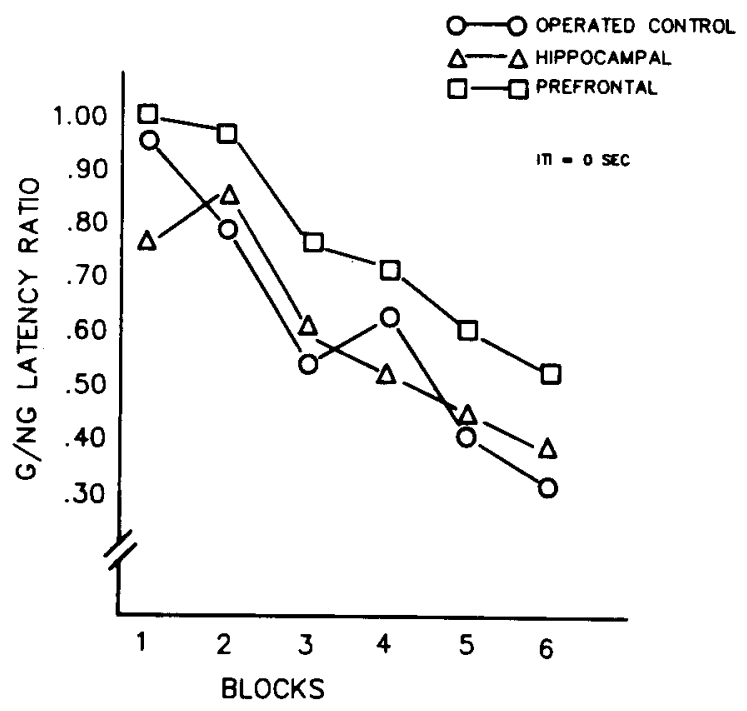

Figure 2. Mean latency ratios and 0-sec ITI of the delayed alternation task, expressed over blocks of two test sessions. 
study were both impaired on the DA task, the respective patterns of deficit were quite different. The hippocampal group had no difficulty learning the alternation rule (see Figure 2) and performed normally at 0 - to 10-sec ITIs (Figure 1). That group's deficit was restricted to performance at 20- to 80-sec ITIs and revealed a failure of episodic memory over relatively long temporal delays. It is noteworthy that rats with lesions to the dorso-medial thalamus were also impaired on this task (Winocur, 1985). These animals were impaired at short and long ITIs, and the extent of their deficit increased with the length of the ITI. It was suggested that dorso-medial thalamic lesions produced an encoding deficit that disrupted both alternation rule learning and memory for trial-related events.

The PFC group, like the thalamic group, was impaired at all ITIs of the DA test. An important difference, however, was that the deficit of the PFC group remained constant over the range of ITIs. The fact that the PFC group's behavior, in relation to the length of the ITI, paralleled that of the $\mathrm{OC}$ group indicates that specific memory function was not selectively affected by prefrontal lesions. The data indicate that the PFC group's deficit was restricted to rule learning and that memory problems related to this deficit were nonspecific in nature.

\section{EXPERIMENT 2}

In Experiment 2, rats with lesions to the hippocampus or prefrontal cortex were compared on a test of conditional discrimination learning (CDL). The test was conducted in an operant chamber outfitted with a panel of lights and two retractable levers. On each trial, a different combination of lights was illuminated just before the appearance of the two levers. The rats were required to learn that illumination on the left side of the panel was the signal for pressing the left lever, whereas illumination on the right side called for a right-lever response.

Previous work is consistent in showing that damage to the prefrontal cortex disrupts performance on tests of conditional learning (Passingham, Myers, Rawlins, Lightfoot, \& Fearn, 1988; Petrides, 1982, 1985). Accordingly, it was predicted that rats with prefrontal lesions would be impaired in the present test. The effects of hippocampal lesions were more difficult to predict. Petrides (1985) found that medial temporal lobe damage that included the hippocampus did not affect CDL in humans. On the other hand, studies of conditional learning in rats have produced conflicting results, with some investigators reporting no effect of hippocampal lesions (e.g., Davidson \& Jarrard, 1989; Jarrard \& Davidson, 1990), and others reporting significant deficits (e.g., Hirsh, 1980; Ross, Orr, Holland, \& Berger, 1984). In view of the conflicting evidence, this experiment was performed to determine whether the present CDL task would discriminate between the effects of prefrontal and hippocampal lesions before introducing delay conditions that would increase demands on specific memory.

\section{Method}

Subjects. Thirty experimentally naive, male Long-Evans rats were obtained from the Trent University Breeding Center and served as subjects. The rats were raised in social cages until they were about 5 months old, at which time they were transferred to individual wire-mesh cages, where they remained throughout the study. Water was available at all times, but food was provided in accordance with experimental conditions.

Apparatus. All testing was conducted in five identical operant chambers $(29 \times 28 \times 26$ in. $)$, each outfitted with two retractable levers, a centrally located visual display panel, and a food well. The display panel consisted of a bank of 2 rows of 3- to 3.5-cp lights, each $1 \mathrm{~cm}$ in diameter and spaced $3 \mathrm{~cm}$ apart. The food well was situated centrally, $5 \mathrm{~cm}$ below the lights and between the levers. A pellet dispenser delivered $45-\mathrm{mg}$ Noyes food pellets to the food well. Three walls and the ceiling of each box were made of Plexiglas. The fourth wall, containing the levers, and so forth, was made of metal, and the floor consisted of steel rods spaced $1.5 \mathrm{~cm}$ apart.

All aspects of testing, including data recording, were controlled by an Apple $\Pi+$ microcomputer, connected to each chamber.

Surgery and Histology. After being housed individually for about 10 days, the rats were assigned to one of three surgical groupshippocampal (HPC), prefrontal (PFC), and operated control (OC). All surgery was conducted in a Johnson-Krieg stereotaxic instrument, with the tooth bar adjusted to a height of $5 \mathrm{~mm}$ above the interaural plane.

Bilateral hippocampal lesions were produced electrolytically by passing a 2-mA DC through a stainless steel electrode insulated except for $0.5 \mathrm{~mm}$ at the tip. Coordinates for hippocampal lesions were $2.2 \mathrm{~mm}$ posterior to bregma, $3.0 \mathrm{~mm}$ below dura, 1.5 and $2.5 \mathrm{~mm}$ lateral to the midline. Current was passed for $15 \mathrm{sec}$ at the $1.5-\mathrm{mm}$ location, and for $20 \mathrm{sec}$ at the $2.5-\mathrm{mm}$ location.

Prefrontal cortex lesions were performed by aspiration. A midline incision was made, and holes, approximately $5 \mathrm{~mm}$ in diameter, were drilled in the anterior wall. With the aid of a surgical microscope, the prefrontal cortex was aspirated through a 20-ga needle.

The rats prepared as operated controls had holes drilled in their skulls, but no brain tissue was destroyed.

After the experiment, all brain-damaged rats were deeply anesthetized with ether and perfused through the heart with physiological saline followed by a $10 \%$ formol saline solution. The brains with hippocampal lesions were frozen and sectioned in the transverse plane throughout the damaged area. The brains with prefrontal lesions were embedded in paraffin before sectioning. All sections were stained with thionin, and the extent of damage was determined by comparisons with the standard atlas of Konig and Klippel (1963).

Procedure. A total of 28 rats survived surgery and participated in the experiment (HPC group, $n=9$; PFC group, $n=9$; OC group, $n=10$ ). Beginning 1 week after surgery, the rats were handled regularly and their body weight was gradually reduced to $80 \%$ of normal. Throughout the experiment, the rats were maintained at a constant weight by being fed about $20 \mathrm{~g}$ of lab chow each day. The rats were introduced to the operant chamber approximately 1 month after surgery. Each rat underwent an extensive three-stage training program before experimental testing began.

Stage 1 . The rats received daily sessions in which they were trained to press both levers according to a CRF schedule. On the 1st day, the rats received some hand-shaping, but, on the following days, they were left to press the levers at their own rates. A rat could obtain a maximum of 160 food pellets per session, distributed equally across left and right leverpresses. When one lever was pressed 80 times, that lever remained in the chamber, but subsequent presses of that lever no longer delivered pellets. Each session in Stage 1 ended when 160 pellets had been delivered, or after a 30-min time limit. The criterion for completing Stage 1 was three consecutive sessions in which the maximum number of pellets was obtained. 
Stage 2. The day after criterion was reached on Stage 1, the rats advanced to Stage 2, where they were trained to become familiar with levers retracting after reinforcement. Each daily session began with both levers present. Pressing either lever produced a food pellet, followed by the retraction of both levers for $10 \mathrm{sec}$. The levers then reappeared and remained in the chamber until a response was performed. The cycle was then repeated. Each lever could be pressed 80 times for reinforcement. When 80 pellets had been obtained with one lever, that lever no longer reappeared, and the session continued with only the other lever appearing and retracting. This procedure served to discourage the build-up of position preferences. The criterion for completing Stage 2 was that the rat must obtain 160 reinforcements in three consecutive 30 -min sessions.

Stage 3. The day after criterion was reached on Stage 2, Stage 3 was instituted to train rats to respond differentially to the presence and absence of light on a given trial. Daily sessions consisted of 100 light and 100 no-light trials, with the order of presentation randomly determined. A light trial began with the illumination of the four middle lights and the simultaneous appearance of both levers. When the rat pressed a lever, a pellet was dispensed, the lights turned off, and the levers retracted. A 10-sec interval followed each light trial. In the light trials, the rats were permitted a maximum of $\mathbf{5 0}$ responses to each lever. When that number was reached, the lever was retracted and did not reappear in the session. In the no-light trials, both levers appeared for $10 \mathrm{sec}$ or until the rat pressed one of the levers. No pellets were provided in the no-light trials. If the rat did not press a lever during the 10 -sec trial, both levers retracted for $10 \mathrm{sec}$. If the rat pressed a lever during a no-light trial, a 30 sec ITI interval followed.

A session was terminated when 200 trials (100 light and 100 nolight) were completed. The criterion for completion of Stage 3 was three consecutive sessions in which the ratio of leverpresses during the light trials to leverpresses during no-light trials was $2: 1$ or better.

Testing. CDL testing began the day after criterion was reached on Stage 3. Each session began with the illumination of the top, bottom, or both lights at one extreme of the panel. The lights, which served as the discriminative stimulus, remained on for $10 \mathrm{sec}$, and their offset was followed immediately with the appearance of both levers. The levers remained in the chamber until a response was performed. A stimulus on the left side was the signal for pressing the left lever, whereas a stimulus on the right side was the signal for a right-lever response. A correct response produced a food pellet and was followed by the retraction of both levers and a 10-sec ITI. An incorrect response led to the withdrawal of the levers, no reinforcement, and a 30-sec ITI.

Each session consisted of 80 trials in which the left lever was correct and 80 trials in which the right lever was correct, the order being randomly determined. One 160-trial session was administered each day for 30 days.

\section{Results and Discussion}

Records were kept of the rats' progress throughout the training program. There were no group differences in reaching criterion in either Stage 1, where rats learned to leverpress according to a CRF schedule, or in Stage 2, where they became familiar with the retraction of levers following responses. There were differences in Stage 3, where rats were trained to relate leverpressing to the presence of a visual stimulus. Here, the $O C$ group reached criterion after an average of 11.0 days of training $(S D=2.16)$, whereas the HPC and PFC groups required $16.7(S D=2.54)$ and $18.1(S D=2.42)$ days, respectively. Both lesion groups differed significantly from the OC group ( $p s<.05$ ), but not from each other.
The results of the CDL test are presented in Figure 3 in terms of the percentage correct responses over the 30 sessions, averaged over six blocks of 5 sessions each. It can be seen that the OC and HPC groups performed similarly throughout testing. The PFC group lagged behind the other groups, and, in fact, the performance of that group did not rise above chance until the fifth block. An ANOVA revealed a significant group $\times$ block interaction $[F(10,25)=3.57, p<.001]$, as well as significant main effects of group $[F(2,25)=12.42, p<.001]$ and block $[F(5,125)=18.25, p<.001]$. A Neuman-Keuls test showed that the significant interaction was due entirely to differences between the PFC and the other groups at Blocks 2-6 inclusive. At no time did the HPC and OC groups differ significantly from each other.

The impaired performance of the PFC group is consistent with other reports of deficits following prefrontal lesions on similar tests of conditional learning (Passingham et al., 1988; Petrides, 1982, 1985). The results provide another example of the disruptive effects of prefrontal lesions on the process of selecting appropriate responses on the basis of discriminative cues. An important feature of the present test is that, although the demands on specific memory were minimal, successful performance did depend on the ability to remember and use nonspecific information. The results provide further evidence of the disruptive effects of prefrontal lesions on tasks that depend on nonspecific memory function for successful performance.

The finding that the HPC and OC groups did not differ on the CDL test is consistent with other reports of nor-

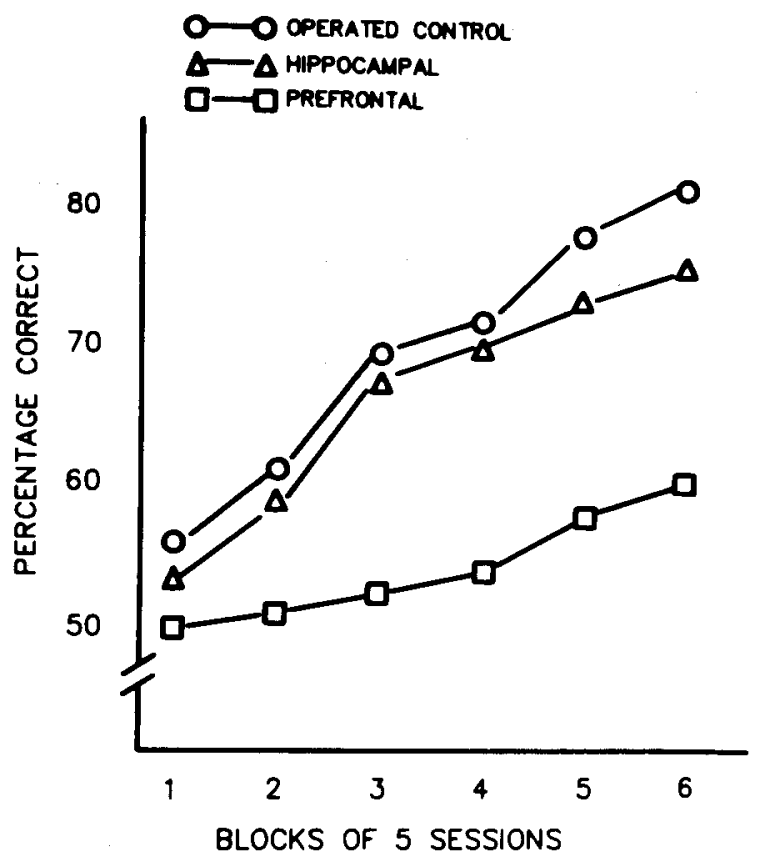

Figure 3. Percentage correct scores in the conditional discrimination learning task of Experiment 2, expressed over six blocks of five test sessions. 
mal conditional learning following hippocampal lesions (Davidson \& Jarrard, 1989; Jarrard \& Davidson, 1990; Petrides, 1985). The reason for the discrepancy between these results and reports of hippocampal deficits on conditional learning tasks is not clear. It is possible that other factors may have contributed to impaired performance in hippocampal groups. For example, Hirsh (1980) found that rats with hippocampal lesions were unable to select specific turning responses on the basis of different discriminative stimuli. However, those tests included a substantial spatial component, and the deficits of hippocampal rats in spatial navigation are well known. In other conditional learning tests (e.g., Ross et al., 1984), trace conditioning procedures forced rats to recall precise information over temporal delays. The importance of the hippocampus in mediating information over time has already been suggested and will be examined further in the next experiment.

\section{EXPERIMENT 3}

The results of Experiment 2 showed that rats with lesions to the prefrontal cortex, but not the hippocampus, were impaired in learning to associate one response with a particular stimulus and a different response with another stimulus. Experiment 3 was performed to assess the effects of varying the interval between presentation of the discriminative stimulus and the opportunity to respond. The purpose was to determine whether challenging memory in this way would differentially affect the performance of the HPC and PFC groups.

A considerable literature suggests that prefrontal lesions do not affect specific memory function (Fuster, 1989; Stuss \& Benson, 1986). The results of Experiment 1 support this contention and, on this basis, the performance of the PFC Group on the CDL task should not be affected by increasing the delay between stimulus and response. However, the possibility that temporal factors contributed to the prefrontal deficit cannot be ruled out. Although the CDL test in Experiment 2 was designed to place minimal demands on specific memory, there was, in fact, a slight delay between the offset of illumination and the appearance of the levers. Further time elapsed while the rat selected a lever and responded. It may be, as Fuster (1989) has argued, that, because of the structure's role in the temporal structuring of behavior, even short delays exacerbate deficits in response planning following prefrontal lesions.

For the present study, a genuine zero-delay condition was built into the CDL test. This was achieved by programming the task so that the visual stimuli and the two levers appeared simultaneously and were present together throughout each trial. Thus, the rat always selected the lever in the presence of the discriminative stimulus. After a fixed number of trials in this condition, the delay between stimulus presentation and the appearance of the levers was increased.
If the PFC group's deficit in Experiment 2 was related to the interval that occurred between the stimulus and response, then removing that interval should result in improved performance. Conversely, lengthening the interval should produce a further deterioration in performance. On the other hand, if the prefrontal deficit resulted primarily from a failure in conditional learning, then varying the interval should have little effect on the PFC group.

With respect to the hippocampus, the results of Experiment 2 are in accord with previous reports that conditional learning is not affected by hippocampal lesions, particularly when demands on specific memory are small (Davidson \& Jarrard, 1989; Squire \& Zola-Morgan, 1985; Winocur, 1985). Thus, a further reduction in the stimulusresponse interval should not affect performance of the HPC group. On the other hand, evidence that animals with hippocampal damage exhibit faster rates of forgetting at relatively long delays (Winocur, 1985, 1990; ZolaMorgan \& Squire, 1985, 1986) leads to the prediction that increasing the interval will contribute to impaired performance in the HPC group.

\section{Method}

The rats used in Experiment 2 also served as subjects in Experiment 2.

About 2 weeks intervened between the end of Experiment 2 and the beginning of Experiment 3. During this time, the rats were maintained on a food-deprivation schedule, with slightly increased daily rations. Before testing, the rats underwent a retraining program that consisted of the three training stages of Experiment 2. The same criteria applied for completing Stages 1-3.

Testing began the day after criterion was reattained on Stage 3. The test sessions were very similar to those of Experiment 2, the only difference being with respect to the interval between the offset of the visual stimuli and the appearance of the two levers. Initially, the rats were presented with a zero-delay condition in which the stimuli and the levers appeared simultaneously and remained together until the rat pressed one of the levers. At that point, the lights turned off, and the levers retracted. If the rats pressed the correct lever, a food pellet was delivered, and a 10-sec ITI followed. A wrong response produced no food and led to a 30-sec ITI. The rats received 15 daily sessions in the zero-delay condition.

The day after the zero-delay testing was completed, a 5-sec delay condition was introduced. Testing was the same for this condition, except that a 5-sec delay intervened between stimulus offset and the appearance of the levers in the chamber. After 15 sessions of 5-sec-delay testing, the rats received 15 sessions in which the delay period was increased to $15 \mathrm{sec}$.

\section{Results and Discussion}

All rats progressed rapidly through each stage of training and there were no group differences in reaching criterion at any stage.

In the zero-delay condition of testing, all groups quickly recovered their performance levels from Experiment 2. In this condition, as in the previous experiment, the HPC and OC groups did not differ from each other, and the PFC group was inferior to the other two. In the delay conditions, the OC and PFC groups displayed slight and comparable declines in performance, relative to their scores 


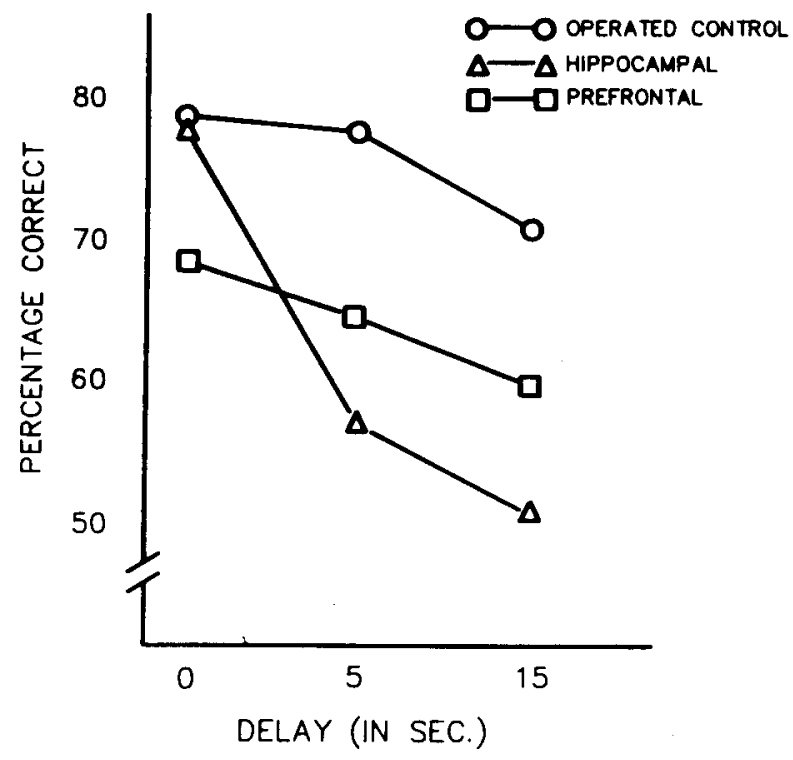

Figure 4. Percentage correct scores in the conditional discrimination learning task of Experiment 3, averaged over the last five sessions in each delay condition.

in the zero-delay condition. In contrast, the HPC group deteriorated dramatically, and, at the 15 -sec delay, its performance did not rise above chance level.

The major results of Experiment 3 are presented in Figure 4 in terms of percentage correct responses averaged over the last five sessions in each delay condition. An ANOVA applied to these data indicated a significant group $X$ delay interaction $[F(4,50)=4.10, p<.01]$, as well as significant main effects of group $[F(2,25)=11.95$, $p<.001]$ and delay $[F(2,50)=16.36, p<.001]$.

In separate analyses, the $\mathrm{OC}$ group was compared with the HPC and PFC groups. Both comparisons yielded significant group effects $(p s<.01)$, but the only significant group $\times$ delay interaction involved the OC and HPC groups $[F(2,34)=24.64, p<.001]$. Post hoc analyses showed that the OC and HPC groups differed significantly in the 5- and 15-sec-delay conditions (both ps $<.001$ ), but not in the zero-delay condition, where their scores were virtually identical. Similar comparisons between the HPC and PFC groups revealed significant differences in all three delays ( $p s<.05$ ), although the differences were not always in the same direction. The HPC group performed better than the PFC group in the zero-delay condition, but worse than the PFC group in the 5- and 15sec-delay conditions.

These results confirm the selective effects of prefrontal lesions on basic conditional discrimination learning. They also show quite clearly that increases in the stimulusresponse interval affected the HPC group more than the other two groups. The rats with hippocampal lesions did not differ from the controls in the zero-delay condition or in Experiment 1 where the delay was minimal, but they displayed disproportionate declines at 5- and 15-sec delays.
The results provide no evidence that the poor performance of the PFC group on this task was related to temporal factors. On the other hand, they confirm that introducing delay periods that increase demands on specific memory can have devastating effects on the behavior of rats with hippocampal lesions. As one might expect, the length of the interval that will affect performance in hippocampally damaged animals varies between tasks. In contrast to the CDL test, where a delay of only $5 \mathrm{sec}$ was sufficient to produce severe impairment in the HPC Group, in the DA test of Experiment 1, rats with hippocampal lesions maintained normal levels of performance at intervals of up to $20 \mathrm{sec}$. One factor that may have contributed to this particular difference is the amount of interference that was operating in the respective tasks. Although high in both cases, the potential for conflict was probably greater in the CDL test, where, on every trial, any one of six stimuli could signal the correct response. It is well established that memory loss associated with hippocampal damage is exaggerated under conditions of high interference (Jarrard, 1975; Winocur, 1979).

\section{Histological Report}

Examples of typical hippocampal and prefrontal lesions are represented diagrammatically in Figure 5 . The lesions closely resembled those reported in the Winocur and Moscovitch (1990) study, in which identical surgical procedures were followed.

The hippocampal lesions destroyed most of the dorsal hippocampus and typically invaded the hippocampal commissure, fimbria, and dorsal fornix areas. There was considerable damage to the overlying corpus callosum and neocortex, but, for the most part, the midline cortex was spared. Occasionally, the lesions extended to the lateral thalamus, but, in all cases, the damage was slight and unilateral. There was no indication that extrahippocampal damage was related to performance in Experiments 2 and 3.

Prefrontal lesions generally extended between 7.5 and $11.0 \mathrm{~mm}$ anterior to the interaural plane. Damage was greatest in dorso-medial regions but also extended to dorso-lateral regions. Slight damage was common in the midline cortex posterior to the genu of the corpus callosum, and, in a few cases, the lesion invaded the corpus callosum.

\section{GENERAL DISCUSSION}

The present results indicate a clear functional dissociation between the hippocampus and prefrontal cortex in learning and memory. Damage to either structure produced severe deficits in tests of delayed alternation and conditional discrimination learning, but the natures of the deficits were quite different. Rats with prefrontal lesions displayed learning deficits in both tests, but their performance was not affected by manipulations that increased demands on specific memory. In contrast, in previous work, rats with hippocampal lesions were normal in learn- 


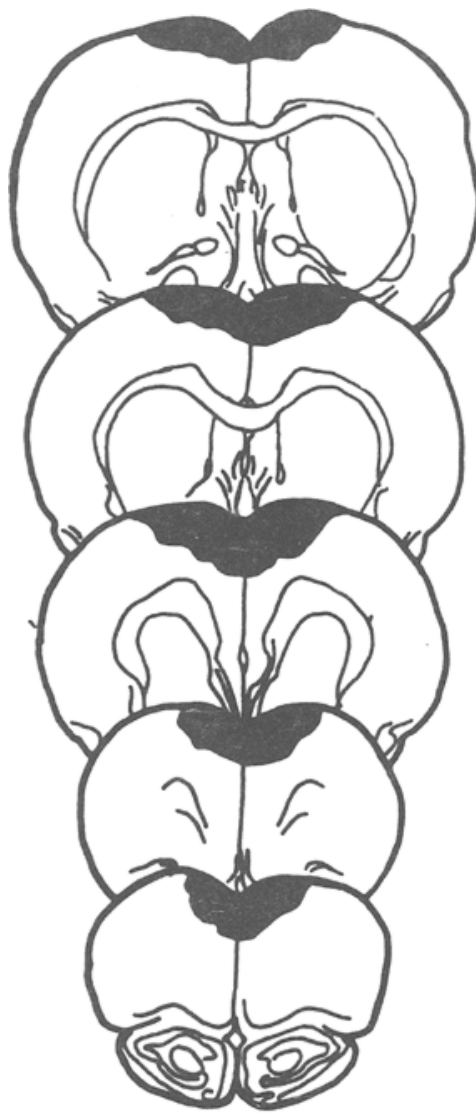

PREFRONTAL
A $7470 \mu$

A $8620 \mu$

A $9650 \mu$

A $10500 \mu$

A $11050 \mu$

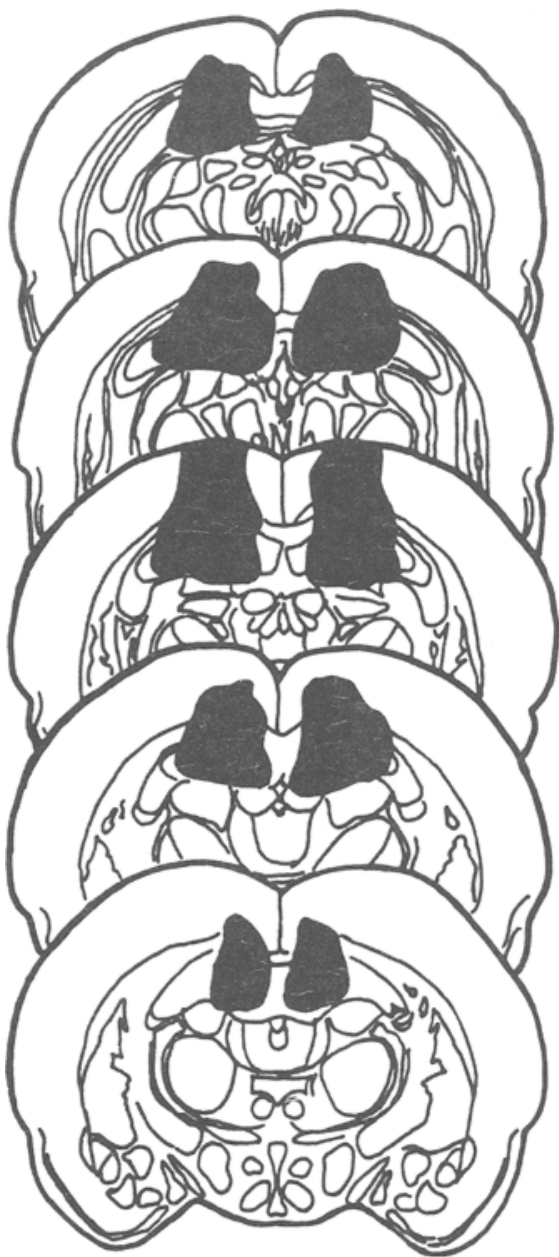

A $2790 \mu$

A $3290 \mu$

A $3750 \mu$

A $4380 \mu$

A $4890 \mu$

Figure 5. Diagrammatic representations of typical prefrontal and dorsal hippocampal lesions.

ing the alternation rule of Experiment 1 and in their performance at relatively short intervals (Winocur, 1985). However, at long intervals, the hippocampal group was severely impaired. Similarly, in Experiments 2 and 3 of the present study, rats with hippocampal lesions did not differ from controls on the basic CDL task, but their performance deteriorated significantly when they were required to recall discriminative stimuli over an extended delay period.

The results contribute to a growing literature that implicates the hippocampus in memory function. They also suggest that the role of this structure in memory may be quite selective and restricted to recalling specific information over the long term. Attempts to understand hippocampal involvement in memory are complicated by the fact that the temporal limits that define long-term memory vary with the task. For example, in the DA task, rats with hippocampal lesions were able to retain and use informa- tion about preceding events for up to $20 \mathrm{sec}$ before performance declined, whereas in the CDL test, the hippocampal group exhibited a marked deficit when they were required to remember a discriminative stimulus for as little as $5 \mathrm{sec}$. In other tests, involving different behavioral measures, rats with hippocampal lesions did not begin to display rapid forgetting until intervals ranging from a few minutes to several weeks had elapsed (Eichenbaum, Parikh, \& Cohen, 1985; Thomson, 1981; Winocur, $1985,1990)$. Comparable results have been obtained in investigations of memory loss in monkeys with hippocampal damage (Zola-Morgan \& Squire, 1985, 1986). In all these studies, the pattern of memory performance following hippocampal damage was essentially the same. Retention was observed to be normal at relatively short delays, but beyond a critical interval that seemed more or less specific for each test, forgetting proceeded at an accelerated rate. There has been little direct study of the variables 
that influence the rate of forgetting in hippocampal subjects, but factors related to level of interference, task difficulty, and spatial demands are undoubtedly important.

The observed pattern of memory loss following hippocampal lesions is consistent with at least two prominent theories of hippocampal function. Rawlins (1985) proposed that the hippocampus acts as a memory store that enables associations to form between temporally displaced events. He supports his argument with considerable evidence that the length of the interval between the events is a critical factor that varies between tasks. The second theory (Squire, Cohen, \& Nadel, 1984) implicates the hippocampus in the process of forming permanent representations of acquired information. This view holds that a variable period of time is required to consolidate information so that it will be available for subsequent retrieval.

The present data do not favor either position, but recent findings from this laboratory offer some support for the consolidation position. In that study (Winocur, 1990), rats with hippocampal lesions exhibited anterograde and retrograde amnesia for a socially acquired food preference. The temporal gradients of memory loss were very similar and suggested that, in this test, the hippocampus was necessary for retaining the preference for more than $48 \mathrm{~h}$ after the learning experience. The results are clearly in line with a consolidation interpretation. Since the paradigm assessed straightforward recall and did not require rats to associate events separated in time, the deficits would not be predicted by Rawlins' (1985) view of the hippocampus as a buffer mechanism.

The results for the PFC groups in the present research reaffirm the structure's involvement in aspects of nonspecific memory. In demonstrating prefrontal deficits in alternation-rule and conditional associative learning, they extend the results of a recent study (Winocur \& Moscovitch, 1990) in which rats with prefrontal lesions were impaired in acquiring a general skill that was essential for performing in complex blind-alley mazes. In these examples, prefrontal rats were selectively impaired in learning and remembering general or nonspecific information, while showing no sign of specific memory loss.

It should be noted that, in the present study, PFC lesions primarily destroyed tissue in the medial prefrontal regions. The possibility remains that more extensive damage to lateral regions and, indeed, larger PFC lesions would have produced greater impairment in the delay conditions. The importance of this point is underscored by the results of a recent study (Fantie \& Kolb, 1990), in which rats with prefrontal lesions were tested in the Morris water maze. The prefrontal rats were impaired on this task, but the magnitude of the deficit was directly related to the lesions' size.

The evidence is mounting that the prefrontal cortex contributes to nonspecific mnemonic function. But given the diversity of learning and memory tasks that depend on the prefrontal cortex, it is difficult to characterize the structure's role in precise terms. Fuster (1989) has proposed that the various effects of lesions to the prefrontal cortex can be related to a fundamental deficit in the temporal organization of behavior. There is considerable support for this position, and the present data, by highlighting deficits in response selection and sequencing, may also be interpreted within the general framework of the theory.

The one aspect of Fuster's (1989) theory that is not supported by the present findings is the emphasis on temporal factors. According to Fuster, "time is probably the single most critical factor determining the involvement of the prefrontal cortex in the synthesis of a novel behavioral structure'" (p. 159). In the present research, the prefrontal deficit did not interact with temporal factors in either the DA or the CDL task. It is especially worth noting that the PFC group was severely impaired in the zero-delay condition of Experiment 3, where the discriminative stimuli and the levers were always simultaneously present. In line with other descriptions of the prefrontal syndrome, the observed deficits could be described in terms of a loss of executive control or an inability to monitor behavior (see Stuss \& Benson, 1986). It does not appear, however, that they can be attributed to a failure in bridging temporally displaced information, as Fuster (1989) has argued.

Although the present data offer no evidence that prefrontal lesions affected the recall of information, they strongly suggest a failure in the utilization of acquired information. In the tests, a rat could select the correct response on a given trial only by relating events of the previous trial (Experiment 1) or the nature of the discriminative stimulus (Experiments 2 and 3 ) to the process of response selection. In other words, successful performance depended on the rat's ability to integrate information from specific cues with present task demands. Other investigators have reported deficits following prefrontal lesions on a variety of tasks that required similar comparisons on individual trials, although, in those studies, it was not always possible to separate effects of temporal delay from the information-processing demands of the task (e.g., Kolb, Sutherland, \& Whishaw, 1983; Milner, 1982; Passingham, 1985).

Shallice (1982) makes the important point that prefrontal damage reliably produces impairment on "nonroutine" tasks, where information must be constantly monitored for appropriate decision making. Deficits are said to be less likely on "routine" tasks, where a response strategy can be formed on the basis of predictable relationships. The disruptive effects of damage to the prefrontal cortex on tests of conditional learning and tasks that require regular behavioral shifts, as in the present research, support this view. Also consistent are reports that prefrontal damage does not affect the ability to form basic stimulusresponse associations or in discrimination learning where particular stimuli are always positive or negative (Milner, 1982; Petrides, 1985).

Shallice (1982) argued that impairments associated with prefrontal damage on nonroutine tasks reflect a fundamental problem with response planning. Moscovitch (1989) offered a similar view in suggesting that such deficits arise 
from a failure in retrieving information for purposes of strategic planning. Others (e.g., Baddeley, 1986; Passingham, 1985) have attributed the behavioral deficits following prefrontal damage to a loss of working memory that interferes with the process of relating cues to the actions being performed.

The various theoretical positions, along with the present data, emphasize the central importance of the prefrontal cortex in an integrative process that relates relevant information to the selection and organization of appropriate behaviors. As such, there are probably more similarities than differences in their essential features. At this point, it would appear that the primary challenge is to determine whether they are sufficiently precise and, at the same time, comprehensive to account for the broad range of deficits produced by prefrontal damage.

\section{REFERENCES}

Badde LeY, A. (1986). Working memory. New York: Oxford University Press.

Cohen, M., SQuire, L. R. (1980). Preserved learning and retention of pattem analyzing skill in amnesia: Dissociation of knowing how and knowing that. Science, 210, 207-209.

DAVIDSON, T. L., \& JARRARD, L. E. (1989). Retention of concurrent conditional discriminations in rats with ibotenate lesions of hippocampus. Psychobiology, 17, 49-60.

Eichengaum, H., Parikh, T., \& Cohen, N. J. (1985). Delayed nonmatched to sample with trial-unique odor stimuli in intact and fornixdamaged rats: A new test for recognition memory and model of temporal-lobe amnesia. Society for Neurosciences, 11, 133. (Abstract)

FANTIE, B. D., \& KolB, B. (1990). An examination of prefrontal lesion size and the effects of cortical grafts on performance of the Morris water task by rats. Psychobiology, 18, 74-80.

FUSTER, J. M. (1989). The prefrontal cortex: Anatomy, physiology, and neuropsychology of the frontal lobe (2nd ed.). New York: Raven.

HiRSH, R. (1980). The hippocampus, conditional operation, and cognition. Physiological Psychology, 8, 175-182.

JARRARD, L. E. (1975). Role of interference and retention by rats with hippocampal lesions. Journal of Comparative \& Physiological Psychology, 89, 400-408.

Jarrard, L. E., \& Davidson, T. L. (1990). Acquisition of concurrent conditional discriminations in rats with ibotenate lesions of hippocampus and of subiculum. Psychobiology, 18, 68-73.

KESNER, R. P. (1984). The neurobiology of memory: Implicit and explicit assumptions. In J. L. McGaugh, G. Lynch, \& N. M. Weinberger (Eds.), Neurobiology of learning and memory (pp. 111-118). New York: Guilford.

KolB, B. (1984). Functions of the frontal cortex of the rat: A comparative review. Brain Research Review, 8, 65-98.

Kolb, B., Sutherland, R. J., \& Whishaw, I. Q. (1983). A comparison of the contributions of the frontal and parietal association cortex to spatial localization in rats. Behavioral Neuroscience, 97, 13-27.

KonIG, J. R., \& KIPPEL, R. E. (1963). The rat brain: A stereotaxic atlas of the forebrain and lower parts of the brain stem. Baltimore, Williams \& Wilkins.

MILNER, B. (1972). Disorders of learning and memory after temporal lobe lesions in man. Clinical Neurosurgery, 19, 421-446.

Milner, B. (1974). Hemispheric specialization: Scope and limit. In F. O. Schmitt \& F. G. Worden (Eds.), The neurosciences: Third study program (pp. 75-89). Cambridge, MA: MIT Press.

MiLNER, B. (1982). Some cognitive effects of frontal-lobe lesions in man. Philosophical Transactions of the Royal Society of London: Series $B, 298,211-226$.

Moscovitch, M. (1989). Confabulation and the frontal system: Strategic vs associative retrieval in neuropsychological theories of memory. In H. L. Roediger III \& F. I. M. Craik (Eds.), Festschriff for Endel Tulving (pp. 133-160). Hillsdale, NJ: Erlbaum.

Olton, D., Becker, J. T., \& Handelmann, G. E. (1979). Hippocampus, space, and memory. Behavioral \& Brain Sciences, 2 , 313-365.

Passingham, R. E. (1985). Memory of monkeys (Macaca mulatta) with lesions in prefrontal cortex. Behavioral Neuroscience, 99, 3-21.

Passingham, R. E., Myers, C., Rawlins, N., Lightfoot, V., \& FEARN, S. (1988). Premotor cortex in the rat. Behavioral Neuroscience, 107, 101-109.

Petrudes, M. (1982). Motor conditional associative leaming after selective prefrontal lesions in the monkey. Behavioral Brain Research, 5, 407-413.

Petrides, M. (1985). Deficits on conditional associative-learning tasks after frontal- and temporal-lobe lesions in man. Neuropsychologia, 23, 601-614.

Pribram, K. H. (1973). The primate frontal cortex-executive of the brain. In K. H. Pribram \& A. R. Luria (Eds.), Psychophysiology of the frontal lobes (pp. 292-314). New York: Academic Press.

Rawlins, J. N. P. (1985). Associations across time: The hippocampus as a temporary memory store. Behavioral \& Brain Sciences, 8 , 479-496.

Ross, R. T., OrR, W. B., Holland, P. C., \& Berger, T. W. (1984). Hippocampectomy disrupts acquisition and retention of learned conditional responding. Behavioral Neuroscience, 2, 211-225.

Shallice, T. (1982). Specific impairments of planning. Philosophical Transactions of the Royal Society of London: Series B, 298, 199-209.

SquiRe, L. R. (1987). Memory and brain. New York: Oxford University Press.

Squire, L. R., Cohen, N. J., \& NAdel, L. (1984). The medial temporal region and memory consolidation: A new hypothesis. In H. Weingartner \& E. Parker (Eds.), Memory consolidation (pp. 185210). Hillsdale, NJ: Erlbaum.

Squire, L. R., \& Zola-Morgan, S. (1985). The neuropsychology of memory: New links between humans and experimental animals. $A n$ nals of the New York Academy of Sciences, 444, 137-149.

Stuss, D. T., \& Benson, D. F. (1986). The frontal lobes. New York: Raven.

Thомson, R. (1981). Rapid forgetting of a spatial habit in rats with hippocampal lesions. Science, 212, 959-960.

WINOCUR, G. (1979). Effects of interference on discrimination learning and recall by rats with hippocampal lesions. Physiology \& Behavior, 22, 339-345.

WinOCUR, G. (1985). The hippocampus and thalamus: Their roles in short- and long-term memory and the effects of interference. Behavioral Brain Research, 16, 135-152.

WINOCUR, G. (1990). Anterograde and retrograde amnesia in rats with dorsal-hippocampal or dorsal-medial thalamic lesions. Behavioral Brain Research, 38, 145-154.

Winocur, G., Moscovitch, M. (1990). Hippocampal and prefontal cortex contributions to learning and memory: Analysis of lesion and aging effects on maze-learning in rats. Behavioral Neuroscience, 104, 544-551.

Zola-Morgan, S., \& Squire, L. R. (1985). Medial temporal lesions in monkeys impair memory on a variety of tasks sensitive to human amnesia. Behavioral Neuroscience, 99, 22-34.

Zola-Morgan, S., \& SQuiRE, L. R. (1986). Memory impairment in monkeys following lesions limited to the hippocampus. Behavioral Neuroscience, 100, 155-160.

(Manuscript received June 14, 1990; revision accepted for publication September 10, 1990.) 